\title{
1 Lower brain pH as a shared endophenotype of psychotic disorders
}

2

3 Hideo Hagihara, $\mathrm{PhD}^{1}$; Vibeke S Catts, $\mathrm{PhD}^{2,3}$; Yuta Katayama, $\mathrm{PhD}^{4}$; Tsuyoshi Takagi, $\mathrm{PhD}^{5,6}$;

4 Freesia L Huang, $\mathrm{PhD}^{7}$; Kuo-Ping Huang, $\mathrm{PhD}^{7}$; Shunsuke Ishii, $\mathrm{PhD}^{6}$; Isabella A Graef, $\mathrm{PhD}^{8}$;

$5 \quad$ Gerald R Crabtree, $\mathrm{MD}, \mathrm{PhD}^{8}$; Keiichi I Nakayama, $\mathrm{MD}, \mathrm{PhD}^{4}$, Cynthia Shannon Weickert, $\mathrm{PhD}^{2,3}$;

6 Tsuyoshi Miyakawa, $\mathrm{PhD}^{1,9}$

7

$8{ }^{1}$ Division of Systems Medical Science, Institute for Comprehensive Medical Science, Fujita Health

9 University, Kutsukake-cho, Toyoake, Aichi 470-1192, Japan

$10{ }^{2}$ Schizophrenia Research Laboratory, Neuroscience Research Australia, Randwick, NSW 2031,

11 Australia

$12{ }^{3}$ School of Psychiatry, University of New South Wales, Sydney NSW 2052, Australia

$13{ }^{4}$ Department of Molecular and Cellular Biology, Medical Institute of Bioregulation, Kyushu

14 University, Higashi-ku, Fukuoka, Fukuoka 812-8582, Japan

$15{ }^{5}$ Institute for Developmental Research, Aichi Human Service Center, Kamiya-cho, Kasugai, Aichi 16 480-0392, Japan

$17 \quad{ }^{6}$ RIKEN Tsukuba Institute, Koyadai, Tsukuba, Ibaraki 305-0074, Japan.

$18{ }^{7}$ Program of Developmental Neurobiology, NICHD, NIH, Bethesda, MD 20892

$19{ }^{8}$ Department of Pathology, Stanford University of Medicine, Stanford, CA 94305-5323

$20{ }^{9}$ Section of Behavior Patterns, Center for Genetic Analysis of Behavior, National Institute for

21 Physiological Sciences, Myodaiji-cho, Okazaki, Aichi 444-8787, Japan 


\section{Correspondence}

24 Tsuyoshi Miyakawa

25 Professor, Division of Systems Medical Science, Institute for Comprehensive Medical Science, 26 Fujita Health University

27 Phone: +81-562-93-9375; Fax: +81-562-92-5382

28 E-mail: miyakawa@fujita-hu.ac.jp

29

\section{Conflict of interest}

31 Dr. Tsuyoshi Miyakawa and Dr. Cynthia Shannon Weickert both receive research grants from

32 Astellas Pharma Inc. Dr. Cynthia Shannon Weickert is a consultant for Lundbeck, Australia Pty 33 Ltd.

34

\section{$35 \quad$ Running title}

36 Lower brain $\mathrm{pH}$ in psychotic disorders 


\section{Abstract}

Lower $\mathrm{pH}$ is a well-replicated finding in the postmortem brains of patients with schizophrenia and

bipolar disorder. Interpretation of the data, however, is controversial as to whether this finding reflects a primary feature of the diseases or is a result of confounding factors such as medication, postmortem interval, and agonal state. To date, systematic investigation of brain $\mathrm{pH}$ has not been undertaken using animal models, which can be studied without confounds inherent in human studies. In the present study, we first confirmed that the brains of patients with schizophrenia and bipolar disorder exhibit lower $\mathrm{pH}$ values by conducting a meta-analysis of existing datasets. We then utilized neurodevelopmental mouse models of psychiatric disorders in order to test the hypothesis that lower brain $\mathrm{pH}$ exists in these brains compared to controls due to the underlying pathophysiology of the disorders. We measured $\mathrm{pH}$, lactate levels, and related metabolite levels in brain homogenates from three mouse models of schizophrenia (Schnurri-2 KO, forebrain-specific calcineurin $\mathrm{KO}$, and neurogranin $\mathrm{KO}$ mice) and one of bipolar disorder (Camk2a $\mathrm{HKO}$ mice), and one of autism spectrum disorders (Chd8 HKO mice). All mice were drug-naïve with the same postmortem interval and agonal state at death. Upon postmortem examination, we observed significantly lower $\mathrm{pH}$ and higher lactate levels in the brains of model mice relative to controls.

53 There was a significant negative correlation between $\mathrm{pH}$ and lactate levels. These results suggest

54 that lower $\mathrm{pH}$ associated with increased lactate levels is a pathophysiology of such diseases rather than mere artifacts. 


\section{Introduction}

58 Schizophrenia, bipolar disorder, and autism spectrum disorders (ASD) are highly heritable

59 psychiatric conditions, with clinical features transcending diagnostic categories ${ }^{1,2}$. Accumulating

60 evidence indicates that some genetic influences ${ }^{3-6}$, gene expression abnormalities ${ }^{7,8}$, and neuronal

61 dysfunctions $^{9,10}$ associated with these conditions overlap, suggesting a common underlying

62 biological basis. However, the shared neurobiological alterations among the three conditions remain

63 largely unknown.

64

65 A number of postmortem studies have indicated that $\mathrm{pH}$ is lower in the brains of patients with

66 schizophrenia and bipolar disorder ${ }^{8,11-19}$. Lower brain $\mathrm{pH}$ has also been observed in patients with

$67 \mathrm{ASD}^{20}$. In general, $\mathrm{pH}$ balance is considered critical for maintaining optimal health, and low $\mathrm{pH}$ has

68 been associated with a number of somatic disorders ${ }^{21-23}$. Therefore, it is reasonable to assume that

69 lower $\mathrm{pH}$ may exert a negative impact on brain function and play a key role in the pathogenesis of

70 various psychiatric disorders. However, lower brain $\mathrm{pH}$ has largely been considered as an

$71 \operatorname{artifact}^{11,24-27}$ rather than a pathophysiology of such disorders ${ }^{13,28}$ for two main reasons. One is that

72 chronic treatment with antipsychotics may affect brain $\mathrm{pH}$ by increasing lactate levels in rats ${ }^{11}$, and

73 most patients with these disorders receive chronic antipsychotics treatment throughout their lives.

74 Another is that the agonistic state experienced before death decreases brain $\mathrm{pH}^{25-27}$ and this state could be different in patients with psychiatric disorders in comparison to controls. In human postmortem studies, it is technically difficult to exclude such confounding factors and to determine whether lower $\mathrm{pH}$ and increased lactate levels are indeed artifacts. 
In the present study, we first confirmed that patients with schizophrenia and bipolar disorder exhibit lower postmortem brain $\mathrm{pH}$ by conducting a meta-analysis of publicly available datasets. We then measured brain $\mathrm{pH}$ in multiple mouse models of psychiatric disorders, which are devoid of such confounding factors, in order to test the hypothesis that lower brain $\mathrm{pH}$ is a pathophysiology or an endophenotype rather than an artifact in a subgroup of psychiatric disorders. We also measured lactate levels, increases in which have frequently been linked to lower $\mathrm{pH}$ in the brains of patients with psychiatric disorders ${ }^{11,13,29}$. To our knowledge, the present study is the first to systematically evaluate $\mathrm{pH}$ and lactate levels in mouse models of psychiatric disorders which eliminate the confounds inherent in the human studies.

For the mouse models of psychiatric disorders, we focused on the ones reported to have neurodevelopmental abnormalities in the brain, a part of which stay at pseudo-immature status ${ }^{30-33}$.

91 Specifically, we measured $\mathrm{pH}$, lactate, and related metabolite levels in the postmortem brains of the

92 following mouse models: schnurri-2 (Shn2) knockout (KO) mice ${ }^{34}$, forebrain-specific calcineurin 93 (Cn) KO mice ${ }^{35-38}$ and neurogranin (Nrgn) KO mice ${ }^{39-41}$ as a model of schizophrenia; mice with

94 heterozygous knockout of the calcium/calmodulin-dependent protein kinase II alpha (Camk2a HKO 95 mice $)^{42,43}$ as a model of bipolar disorder; , and mice with heterozygous knockout of the long isoform 96 of chromodomain helicase DNA-binding protein 8 (Chd8 HKO mice) ${ }^{33}$ as a model of ASD.

97 These mouse strains have mutations in the genes implicated in the respective disorders and exhibit 98 molecular and behavioral abnormalities relevant to each condition, indicating good construct and 
99 face validities, respectively (as described in detail in Materials and Methods).

100

\section{Materials and Methods}

\section{Human data}

103 Nine publicly available datasets were utilized in the present study (Supplementary Table 1): four 104 schizophrenia datasets (GSE17612, GSE21935, GSE21138; NSWBTRC-SC ${ }^{44}$ ), one bipolar 105 disorder dataset (GSE5392), and three combined schizophrenia and bipolar disorder datasets 106 (Stanley Medical Research Institute [SMRI] Collection A, SMRI Collection C, GSE35977, 107 GSE53987). We obtained data regarding postmortem interval and age from these studies and data regarding medication from SMRI Collection A and SMRI Collection C.

\section{Animals}

111 We measured $\mathrm{pH}$, lactate, and related metabolite levels in Shn2 KO mice ${ }^{34}(\mathrm{n}=5,6$ [controls,

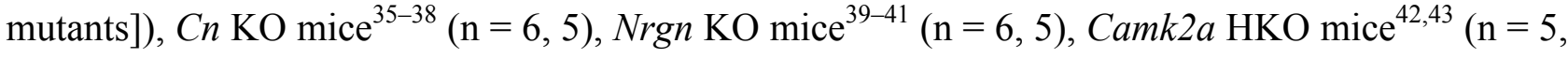

113 5) and Chd8 HKO mice ${ }^{33}(\mathrm{n}=5,5)$, and their corresponding control mice. Both male and female

114 mice were used in the present study, as no difference in $\mathrm{pH}$ between genders has been observed ${ }^{45}$.

115 All mice were between 19 and 45 weeks of age, and no significant difference in age was observed 116 between controls and mutants within each strain. All animal experiments were approved by the 117 Institutional Animal Care and Use Committee of Fujita Health University, based on the Law for the 118 Humane Treatment and Management of Animals and the Standards Relating to the Care and 119 Management of Laboratory Animals and Relief of Pain. Every effort was made to minimize the 
number of animals used.

122 Shn2 was originally identified as a nuclear factor- $\kappa \mathrm{B}(\mathrm{NF}-\kappa \mathrm{B})$ site-binding protein that tightly binds 123 to the enhancers of major histocompatibility complex (MHC) class I genes and acts as an 124 endogenous inhibitor of NF- $\kappa \mathrm{B} .^{46}$ Its deficiencies in Shn2 may cause mild chronic inflammation in 125 the brain and confer molecular, neuronal, and behavioral phenotypes relevant to schizophrenia in 126 mice $^{34}$. Genome-wide association studies (GWASs) have identified a number of single nucleotide 127 polymorphisms (SNPs) in the MHC region associated with schizophrenia ${ }^{47-49}$. Shn2 KO mice 128 exhibit multiple abnormal behaviors related to schizophrenia, including increased locomotor activity, deficits in working memory, abnormal social behavior and impaired prepulse inhibition, which are commonly observed in $\mathrm{Cn} \mathrm{KO}$ mice ${ }^{35,36}$ and Nrgn $\mathrm{KO}$ mice ${ }^{39-41}$ as well.

132 Calcineurin $(\mathrm{Cn})$ is a calcium-dependent protein phosphatase and has been implicated in synaptic 133 plasticity ${ }^{50}$. $C N$ has been reported to be associated with schizophrenia ${ }^{51-53}$, and altered expression of 134 calcineurin has been observed in the postmortem brains of patients with schizophrenia ${ }^{54,55}$. 135 Forebrain-specific $C n \mathrm{KO}$ mice exhibit behavioral and cognitive abnormalities related to 136 schizophrenia ${ }^{35,36}$. Deficits in synaptic transmission in the frontal cortex have been suggested to be 137 the underlying mechanism of working memory impairment in these mice ${ }^{38}$. In addition, $C n$ KO 138 mice exhibit disruption in ripple-associated information processing in the hippocampal CA1, which 139 is implicated in cognitive impairments associated with schizophrenia ${ }^{37}$. 
141 Neurogranin (Nrgn) is a calmodulin-binding protein that modulates activity of the Camk2 protein 142 downstream of $N$-methyl-d-aspartic acid (NMDA) receptors, and is implicated in synaptic 143 plasticity $^{39}$. GWAS revealed significant association with SNPs located upstream of the $N R G N^{49}$, a 144 finding recently confirmed by a large-scale $\mathrm{GWAS}^{56}$, strongly suggesting that $N R G N$ is a 145 susceptibility gene for schizophrenia. Nrgn KO mice exhibit behavioral phenotypes related to 146 schizophrenia ${ }^{39-41}$.

148 Camk2 is a major downstream molecule of the NMDA receptor and is thought to play an essential 149 role in synaptic plasticity. A recent study demonstrated genetic association of $C A M K 2 A$ with 150 bipolar disorder ${ }^{57}$, and decreased mRNA expression has been observed in the frontal cortex of 151 patients with bipolar disorder ${ }^{58}$. In addition, the Camk2a gene was identified as one of the top 152 candidate genes for bipolar disorder by a meta-analysis that integrated genetic and genomic data 153 from both human and animal studies $^{59}$. At cellular level, neuronal hyperexcitability, which we 154 previously detected in the hippocampal granule cells of Camk2a HKO mice ${ }^{42}$, was also found in the 155 granule cell-like neurons differentiated from induced pluripotent stem cells (iPSCs) derived from 156 patients with bipolar disorder ${ }^{60}$. Camk2a HKO mice exhibit abnormal behaviors, such as deficits in 157 social activity and working memory, which are analogous to those in patients with bipolar 158 disorder/schizophrenia ${ }^{42}$. In addition, these mutant mice exhibit infradian cyclic activity levels, 159 which may reflect infradian oscillation of mood substantially observed in patients with bipolar 160 disorder ${ }^{43}$. These findings suggest that Camk2a HKO mice have construct and face validity as a 161 model of psychiatric disorders, especially of bipolar disorder. 
163 Chd8, a member of the chromodomain helicase DNA-binding family of proteins, is known to act as 164 a chromatin-remodeling factor. Recent exome sequencing analyses have identified a number of de 165 novo mutations in a variety of genes in individuals with ASD, further revealing that $C H D 8$ is the

166 most frequently affected gene ${ }^{61-64}$. Chd8 HKO mice exhibit behavioral abnormalities reminiscent of 167 ASD in humans, including increased anxiety, increased persistence and abnormal social 168 interaction $^{33}$. Chd8 deficiency induces aberrant activation of RE1 silencing transcription factor 169 (REST), a molecular brake of neuronal development, resulting in neurodevelopment abnormalities 170 in mice $^{33}$.

171

172 Collectively, these findings indicate that the mouse models used in the present study exhibit good 173 construct and face validities for their respective disorders.

\section{Measurement of pH}

Mice were sacrificed by cervical dislocation followed by decapitation, following which whole

177 brains were removed. The brains were immediately frozen in liquid nitrogen and stored at $-80^{\circ} \mathrm{C}$ 178 until use. We measured brain $\mathrm{pH}$ basically as previously described ${ }^{11}$. Briefly, the brains were 179 homogenized using the tissue homogenizer attached with a conical pestle in ice-cold distilled $\mathrm{H}_{2} \mathrm{O}$ 180 (5 mL per $500 \mathrm{mg}$ of tissue). The $\mathrm{pH}$ was measured using a $\mathrm{pH}$ meter (LAQUA F-72, Horiba 181 Scientific, Kyoto, Japan) after a three-point calibration at $\mathrm{pH} 4.0, \mathrm{pH} 7.0$ and $\mathrm{pH}$ 9.0. The $\mathrm{pH}$ of the 182 samples from control and mutant mice were read in triplicate for each sample. After $\mathrm{pH}$ 
183 measurement, homogenates were immediately frozen and stored at $-80^{\circ} \mathrm{C}$ until required for further 184 analyses.

\section{Lactate and glucose measurements}

187 The concentration of lactate in the brain homogenates was determined using a multi-assay analyzer 188 (GM7 MicroStat; Analox Instruments, London, UK) according to manufacturer's instructions. In 189 our prior tests using several samples, we loaded $5 \mu 1,10 \mu 1$ and $20 \mu 1$ of supernatants to the 190 instrument, observing that the measurements increased linearly in a volume-dependent manner $\left(r^{2}>\right.$ 191 0.99). Based on these results, we used $20 \mu \mathrm{l}$ of supernatants for each sample for lactate measurements. Likewise, glucose concentrations in $20 \mu 1$ supernatant samples were determined using a multi-assay analyzer following calibration with $10 \mathrm{mmol} / \mathrm{ml}$ glucose standard solution. To normalize the effects of differences among strains, such as genetic background and age, $z$-scores for $\mathrm{pH}$ and lactate levels were calculated within each strain and used for the correlation analysis.

\section{Pyruvate measurement}

198 Pyruvate concentrations in $20 \mu \mathrm{l}$ supernatant samples were determined using a pyruvate assay kit 199 (BioVision, Mountain View, CA, USA). The fluorescence intensities were measured using a 200 microplate reader equipped with a spectrofluorometer (ARVO X, PerkinElmer).

\section{Adenosine diphosphate/adenosine triphosphate (ADP/ATP) ratio}

203 An ADP/ATP Ratio Assay Kit (BioVision) was used to measure the ADP and ATP concentrations 
204 according to the manufacturer's instructions.

\section{Bioinformatics analysis of transcriptome data}

207 We used the following mouse brain transcriptome data: frontal cortex and hippocampal dentate 208 gyrus of Shn2 KO mice (microarray) ${ }^{34}$, hippocampal dentate gyrus of Camk2a HKO mice 209 (microarray) ${ }^{65}$, and whole brains of Chd8 HKO mice (RNA-sequencing) ${ }^{33}$. Gene expression 210 patterns of the frontal cortex of Camk2a HKO mice $(\mathrm{n}=6,6)$ and hippocampal DG of $C n \mathrm{KO}$ mice $211(\mathrm{n}=6,6)$ were analyzed via microarray (Mouse Genome 4302.0 Array; Affymetrix, Santa Clara, 212 CA, USA), as previously described ${ }^{34}$. Gene expression patterns of the frontal cortex and 213 hippocampal DG of $\operatorname{Nrgn} \operatorname{KO}$ mice $(\mathrm{n}=5,5)$ were analyzed via RNA-sequencing using the HiSeq 214 platform basically according to the manufacturer's instructions (Illumina, San Diego, CA, USA). 215 Genes with an absolute fold change $>1.2$ and a $t$-test $P$-value $<0.05$ (mutants vs. controls; without 216 correction for multiple testing) were imported into the bioinformatics tool BaseSpace (Illumina), 217 with which the gene expression data obtained from different platforms can be matched. Genes with 218 altered expression in at least four out of the eight datasets (yielding 80 features; Supplementary 219 Table 2) were selected based on the criteria of the BaseSpace tool and assessed for enrichment in 220 biological themes using the DAVID functional annotation clustering tool, ADGO, and GOToolBox, 221 in which the default feature listings and algorithm settings were used.

Results

\section{Meta-analysis of human brain pH studies}


225 We first re-evaluated the results of postmortem studies of brain $\mathrm{pH}$ in patients with schizophrenia 226 and bipolar disorder by conducting a meta-analysis of publicly available datasets. We searched the 227 National Center for Biotechnology Information Gene Expression Omnibus (NCBI GEO), 228 ArrayExpress, and Stanley Medical Research Institute (SMRI) databases, and found nine studies 229 that included individual brain $\mathrm{pH}$ data from patients with schizophrenia, bipolar disorder, or both as 230 well as from healthy control participants (Supplementary Table 1). A two-way analysis of variance 231 (ANOVA) revealed a significant effect of condition $\left(\mathrm{F}_{2,645}=3.35, P=3.09 \times 10^{-10}\right)$ and study $\left(\mathrm{F}_{8,645}\right.$ $\left.232=10.00, P=2.00 \times 10^{-16}\right)$ as well as between the two factors $\left(\mathrm{F}_{11,645}=47.66, P=0.043\right)($ Figure 1$)$.

233 Post hoc comparisons with Tukey's honest significant difference test indicated a lower brain $\mathrm{pH}$ in 234 both patients with schizophrenia $\left(P<1.0 \times 10^{-7}\right)$ and bipolar disorder $(P=0.00036)$ compared to 235 healthy controls, and no significant difference between the two conditions $(P=0.56)$. The results of 236 our meta-analysis therefore support the finding of lower brain $\mathrm{pH}$ in patients with schizophrenia and 237 bipolar disorder.

Brain $\mathrm{pH}$ was not correlated with lifetime use of antipsychotics (measured as fluphenazine equivalents) in a schizophrenia group (Pearson's $r=-0.27, p=0.12)$, a bipolar disorder group $(r=$ $241-0.27, P=0.13)$, or a group including both conditions $(r=-0.15, P=0.23)$ from the SMRI 242 Collection A cohort (Supplementary Figure 1a). No correlation between $\mathrm{pH}$ and antipsychotics was 243 replicated in a separate cohort from SMRI Collection C (schizophrenia: $r=0.16, P=0.58$; bipolar 244 disorder: $r=0.036, P=0.90$; both: $r=0.090, P=0.64)$ (Supplementary Figure $1 \mathrm{~b}$ ). These suggest 245 that antipsychotics treatment may not affect $\mathrm{pH}$ in the postmortem brains of patients with 
246 schizophrenia and bipolar disorder. On the other hand, in the combined data, $\mathrm{pH}$ was positively

247 correlated with postmortem interval $(r=0.13, P=0.0010$; Supplementary Figure 1c) and negatively

248 correlated with age ( $r=-0.13, P=0.00092$; Supplementary Figure 1d), suggesting that these factors

249 may contribute to the changes in the $\mathrm{pH}$ of postmortem brains.

250

251 Lower pH and increased lactate levels in the postmortem brain of mouse models of 252 schizophrenia, bipolar disorder, and ASD

253 The confounding factors identified in previous studies ${ }^{11,26}$ are beyond investigator's control in 254 human postmortem brain studies. We therefore measured $\mathrm{pH}$ and lactate levels in the brains of 255 mouse models of schizophrenia (Shn2 KO, Cn KO, Nrgn KO mice), bipolar disorder (Camk2a 256 HKO mice), and ASD (Chd8 HKO mice). All the mice used were drug-naïve and sacrificed by 257 cervical dislocation (controling for agonal state differences). The removed brains were snap-frozen 258 within a few minutes (controling for postmortem interval differences). Brain pH was significantly 259 lower in all five mutant strains examined relative to the corresponding controls (Shn2 KO, $7.17 \pm$ 2600.0060 , controls [Con], 7.20 $\pm 0.056, P=0.0083 ; C n \mathrm{KO}, 7.08 \pm 0.0057$, Con, $7.13 \pm 0.0080, P=$ $2610.0014 ;$ Nrgn KO, $7.10 \pm 0.017$, Con, $7.16 \pm 0.0080, P=0.0090 ;$ Camk2a HKO, $7.14 \pm 0.0093$, 262 Con, 7.21 \pm 0.0090, $P=0.0014 ;$ Chd8 HKO, $7.08 \pm 0.0066$, Con, $7.12 \pm 0.0031, P=0.00080)$ 263 (Figure 2a).

265 Significantly higher levels of lactate were observed in the postmortem brains of all mutant mice 266 strains compared to corresponding controls $(S h n 2 \mathrm{KO}, 2.98 \pm 0.080 \mathrm{mM}$, Con, $2.55 \pm 0.076 \mathrm{mM}, P$ 
$267=0.0038 ;$ Cn KO, $3.24 \pm 0.051 \mathrm{mM}$, Con, $2.90 \pm 0.073 \mathrm{mM}, P=0.0052 ;$ Nrgn KO, $2.98 \pm 0.11$

$268 \mathrm{mM}$, Con, $2.58 \pm 0.054 \mathrm{mM}, P=0.0080 ;$ Camk2a HKO, $2.86 \pm 0.024 \mathrm{mM}$, Con, $2.58 \pm 0.037 \mathrm{mM}$,

$269 P=0.00024 ;$ Chd8 HKO, $3.04 \pm 0.081 \mathrm{mM}$, Con, $2.58 \pm 0.086 \mathrm{mM}, P=0.0046$; Figure 2b).

270 Analysis of the combined data expressed as the $z$-score revealed that $\mathrm{pH}$ was significantly

271 negatively correlated with lactate levels (Pearson's $r=-0.65, P=1.19 \times 10^{-7}$; Figure $2 \mathrm{c}$ ).

272

273 Lactate is formed from pyruvate during glycolysis. We therefore measured pyruvate levels in 274 mutant mouse brains and observed that levels were significantly increased in Shn2 KO $(P=0.011)$, $275 C n \mathrm{KO}(P=0.046), \operatorname{Nrgn} \mathrm{KO}(P=0.011)$ and $C h d 8 \mathrm{HKO}$ mice $(P=0.0036)$ and showed increased 276 tendency in Camk2a HKO mice $(P=0.068)$ (Figure 2d). Glucose levels remained unchanged in 277 mutant mice relative to controls (Figure 2e), suggesting glucose supply/demand ratio in the brain 278 may be comparable in these mouse models. The ADP/ATP ratio was decreased in $\mathrm{Nrgn} \mathrm{KO}$ mice $(P$ $279=0.035)$ and increased in $C h d 8 \mathrm{HKO}$ mice $(P=0.047)$ (Figure 2f), suggesting a contrasting energy 280 consumption ratio in mouse models of schizophrenia and ASD.

282 We then analyzed transcriptome data (Supplementary Table 2) in order to investigate the potential 283 underlying molecular mechanisms of increased lactate levels in mutant mouse brains. The 284 transcriptome data from five mouse strains revealed an enrichment in Wnt- and epidermal growth 285 factor (EGF)-related pathways when analyzed with DAVID software (Supplementary Table 3). 286 Enrichment in Wnt-related pathways was replicated in the analyses using other bioinformatics tools 287 (ADGO and GOToolBox) using different statistical methods (Supplementary Table 3). 
289 Since lactate is produced via glycolytic pathways in astrocytes in the brain ${ }^{66}$, we analyzed the 290 transcriptome data of mutant mice with particular focus on glycolysis-related genes (Gene Ontology

291 Consortium database), as well as those related to pyruvate metabolism. The results of the targeted 292 gene expression analyses suggest that elevated glycolysis and pyruvate metabolism shifting toward 293 lactate synthesis occurs in the brains of mutant mice, especially in Shn2 KO and Camk2a HKO 294 mice (Supplementary Table 4; Supplementary Figure 2).

\section{Discussion}

297 In the present study, we confirmed lower $\mathrm{pH}$ in the postmortem brains of patients with 298 schizophrenia and bipolar disorder by conducting a meta-analysis of existing datasets. Lower $\mathrm{pH}$ 299 was also observed in five different mouse models of psychiatric disorders, all of which were 300 drug-naïve and were controlled for other confounding factors, such as agonal state and postmortem 301 interval. We also observed increased lactate levels in the brains of mutant mice, as well as a highly 302 significant negative correlation between $\mathrm{pH}$ and lactate levels, which is consistent with the findings 303 of previous human postmortem studies ${ }^{11}$. These results suggest that lower $\mathrm{pH}$ and increased lactate 304 levels represent components of the underlying pathophysiology of the diseases rather than mere 305 artifacts.

307 Researches have revealed that brain acidosis influences a number of brain functions, such as anxiety, 308 mood, and cognition ${ }^{67}$. Acidosis may affect the structure and function of several types of brain cells, 
309 including the electrophysiological functioning of GABAergic neurons ${ }^{68}$ and morphological

310 properties of oligodendrocytes ${ }^{69}$. Alterations in these types of cells have been well-documented in

311 the brains of patients with schizophrenia, bipolar disorder, and $\mathrm{ASD}^{70,71}$ and may underlie some of

312 the cognitive deficits associated with these disorders. Deficits in GABAergic neurons and

313 oligodendrocytes have been identified in the mouse models of the disorders, including Shn2 KO

314 mice $^{30,34}$. Brain acidosis may therefore be associated with deficits in such cell types in 315 schizophrenia, bipolar disorder, and ASD.

317 A previous study indicated that chronic treatment with antipsychotics increases lactate levels in the 318 rat cerebral cortex ${ }^{11}$, suggesting that such increases may be medication-related. The authors of the 319 report, however, found no significant correlation between lactate levels and history of antipsychotic 320 use (which was represented by chlorpromazine equivalents) in the postmortem brains of patients 321 with schizophrenia ${ }^{11}$. In addition, increased lactate levels have been observed in the anterior 322 cingulate of medication-free patients with bipolar disorder in in vivo spectroscopic imaging 323 studies $^{72}$. Furthermore, studies utilizing animal models of psychiatric disorders-including the 324 current study-have identified increased lactate levels in mutant mouse brains ${ }^{73}$. In addition, 325 increased lactate levels were associated with lower $\mathrm{pH}$ in the brains of mutant mice, consistent with 326 findings from previous studies on patients with schizophrenia ${ }^{11,13}$. Lower brain $\mathrm{pH}$ has also been 327 observed in the medication-free patients with bipolar disorder ${ }^{28}$. Although it remains possible that 328 antipsychotic treatment increases lactate levels and lowers $\mathrm{pH}$ in the brain, the aforementioned 329 findings suggest that such changes may occur as primary features of schizophrenia and bipolar 
disorder.

Interestingly, we observed that Wnt- and EGF-related pathways, which are highly implicated in somatic and brain cancers ${ }^{74}$, are enriched in the genes whose expressions were altered among the

334 five mutant mouse strains. It is known that cancer cells display high rates of glycolysis, resulting in high lactate and pyruvate levels, even in normoxia ${ }^{75}$; this phenomenon has been referred to as the 336 Warburg effect. Genes whose expression is known to positively regulate the Warburg effect, such as $H k 2^{76}, H i f l a^{75}$, and $P f k f b 3,{ }^{77}$ were increased in the brains of any of mouse models examined in the present study, while expression of Prkaal, a negative regulator of the Warburg effect $^{78}$, was decreased (Supplementary Table 2). These findings raise the possibility that elevated glycolysis underlies the increased lactate and pyruvate levels in the brains of the mouse models of schizophrenia, bipolar disorder, and ASD. The results of the targeted gene expression analyses conducted in the present study also support the hypothesis. Glycolysis is also stimulated by the 343 uptake of glutamate in astrocytes following neuronal excitation ${ }^{79}$. Dysregulation of the 344 excitation-inhibition balance has been proposed as a candidate cause of schizophrenia, bipolar 345 disorder, and $\mathrm{ASD}^{80,81}$. A shift in the balance towards excitation would result in increased energy 346 expenditure and may lead to increased glycolysis. Indeed, Shn2 KO mice exhibit higher glutamate 347 levels in the hippocampus ${ }^{34}$. In vivo metabolite measurements have indicated that increased 348 glycolysis occurs in the brains of patients with bipolar disorder ${ }^{29,72}$, while gene ontology analysis of microarray data has indicated that decreased glycolysis occurs in the brains of patients with schizophrenia $^{13}$. Further studies are required to determine whether altered glycolysis rate is 
associated with increased lactate levels.

353 It has been indicted that lactate levels in the mouse brain rapidly increase after at least 1 min of 354 decapitation as compared to in vivo fixation by focused microwave irradiation, which is regarded as 355 a consequence of enhanced glycolysis under oxygen-deprived conditions ${ }^{82}$. While the current 356 findings may differ from those obtained under physiological conditions, they may reflect functional 357 changes, such as the activation of astrocytes ${ }^{34,40}$, which represent the main source of lactate 358 production in the brain.

Brain $\mathrm{pH}$ is associated with notable changes in gene expression ${ }^{16,26,45,83}$ and has hence been considered as a confound for investigating changes in gene expression related to the pathophysiology of psychiatric disorders. Therefore, substantial effort has been made to match the tissue $\mathrm{pH}$ between patients and controls. Given that lower brain $\mathrm{pH}$ is a pathophysiology of certain conditions, $\mathrm{pH}$-dependent changes in gene expression would not be negligible when attempting to elucidate the molecular basis of the conditions. It has been known that gene expression patterns are partially similar across diseases such as schizophrenia, bipolar disorder, and $\mathrm{ASD}^{7,8}$. Lower $\mathrm{pH}$ may underlie the similarities of gene expression patterns. Thus, $\mathrm{pH}$ may be an important factor in the elucidation of molecular alternations in the brains of patients with these psychiatric conditions.

\section{Acknowledgments}

371 We thank Wakako Hasegawa, Yumiko Mobayashi, Misako Murai, Tamaki Murakami, Miwa 
372 Takeuchi, Satoko Hattori and Aki Miyakawa, Fujita Health University, for their technical support in

373 this study, and Yuki Sugiura, Keio University, for helpful discussion. This work was supported by 374 JSPS Grant-in-Aid for Scientific Research on Innovative Areas Grant Number 25116526, 375 15H01297, JSPS KAKENHI Grant Number 25242078, and AMED Strategic Research Program for 376 Brain Sciences.

\section{Author contributions}

379 Conceived and designed the experiments: HH and TM. Contributed materials: VC, YK, TT, FH, KH, SI, IG, GC, KN and CW. Analyzed the data: HH and TM.

\section{Conflict of interest}

Dr. Tsuyoshi Miyakawa and Dr. Cynthia Shannon Weickert both receive research grants from 384 Astellas Pharma Inc. Dr. Cynthia Shannon Weickert is a consultant for Lundbeck, Australia Pty Ltd. 385 Other authors have no conflict of interests to declare.

\section{$387 \quad$ References}

3881 Hyman SE. The Diagnosis of Mental Disorders: The Problem of Reification. Annu Rev 389 Clin Psychol 2010; 6: 155-179.

3902 Insel T, Cuthbert B, Garvey M, Heinssen R, Pine DS, Quinn K et al. Research Domain 391 Criteria (RDoC): Toward a New Classification Framework for Research on Mental Disorders. Am J 392 Psychiatry 2010; 167: 748-751.

3933 Carroll LS, Owen MJ. Genetic overlap between autism, schizophrenia and bipolar 394 disorder. Genome Med 2009; 1: 102.

3954 Cross-Disorder Group of the Psychiatric Genomics Consortium. Genetic relationship 
between five psychiatric disorders estimated from genome-wide SNPs. Nat Genet 2013; 45: 984

397994.

3985 Cross-Disorder Group of the Psychiatric Genomics ConsortiumConsortium. Identification 399 of risk loci with shared effects on five major psychiatric disorders: a genome-wide analysis. The $400 \quad$ Lancet 2013; 381: 1371-1379.

4016 Lotan A, Fenckova M, Bralten J, Alttoa A, Dixson L, Williams RW et al.

402 Neuroinformatic analyses of common and distinct genetic components associated with major 403 neuropsychiatric disorders. Neurogenomics 2014; 8: 331.

4047 Ellis SE, Panitch R, West AB, Arking DE. Transcriptome analysis of cortical tissue 405 reveals shared sets of downregulated genes in autism and schizophrenia. Transl Psychiatry 2016; 6: $406 \mathrm{e} 817$.

4078 Shao L, Vawter MP. Shared gene expression alterations in schizophrenia and bipolar 408 disorder. Biol Psychiatry 2008; 64: 89-97.

4099 Goodkind M, Eickhoff SB, Oathes DJ, et al. Identification of a common neurobiological 410 substrate for mental illness. JAMA Psychiatry 2015; 72: 305-315.

$41110 \quad$ Yahata N, Morimoto J, Hashimoto R, Lisi G, Shibata K, Kawakubo Y et al. A small 412 number of abnormal brain connections predicts adult autism spectrum disorder. Nat Commun 2016; $413 \quad$ 7: 11254.

41411 Halim ND, Lipska BK, Hyde TM, Deep-Soboslay A, Saylor EM, Herman M et al. 415 Increased lactate levels and reduced $\mathrm{pH}$ in postmortem brains of schizophrenics: medication 416 confounds. J Neurosci Methods 2008; 169: 208-213.

41712 Guillozet-Bongaarts AL, Hyde TM, Dalley RA, Hawrylycz MJ, Henry A, Hof PR et al. 418 Altered gene expression in the dorsolateral prefrontal cortex of individuals with schizophrenia. Mol 419 Psychiatry 2014; 19: 478-485.

$42013 \quad$ Prabakaran S, Swatton JE, Ryan MM, Huffaker SJ, Huang JT-J, Griffin JL et al. 421 Mitochondrial dysfunction in schizophrenia: evidence for compromised brain metabolism and 422 oxidative stress. Mol Psychiatry 2004; 9: 684-697, 643.

42314 Lipska BK, Deep-Soboslay A, Weickert CS, Hyde TM, Martin CE, Herman MM et al. 424 Critical factors in gene expression in postmortem human brain: focus on studies in schizophrenia. 425 Biol Psychiatry 2006; 60: 650-658.

42615 Torrey EF, Barci BM, Webster MJ, Bartko JJ, Meador-Woodruff JH, Knable MB.

427 Neurochemical markers for schizophrenia, bipolar disorder, and major depression in postmortem 
brains. Biol Psychiatry 2005; 57: 252-260.

$42916 \quad$ Iwamoto K, Bundo M, Kato T. Altered expression of mitochondria-related genes in 430 postmortem brains of patients with bipolar disorder or schizophrenia, as revealed by large-scale 431 DNA microarray analysis. Hum Mol Genet 2005; 14: 241-253.

43217 Ryan MM, Lockstone HE, Huffaker SJ, Wayland MT, Webster MJ, Bahn S. Gene 433 expression analysis of bipolar disorder reveals downregulation of the ubiquitin cycle and alterations 434 in synaptic genes. Mol Psychiatry 2006; 11: 965-978.

43518 Sun X, Wang J-F, Tseng M, Young LT. Downregulation in components of the 436 mitochondrial electron transport chain in the postmortem frontal cortex of subjects with bipolar 437 disorder. J Psychiatry Neurosci 2006; 31: 189-196.

43819 Mistry M, Gillis J, Pavlidis P. Meta-analysis of gene coexpression networks in the 439 post-mortem prefrontal cortex of patients with schizophrenia and unaffected controls. $B M C$ $440 \quad$ Neurosci 2013; 14: 105.

$44120 \quad$ Young A, Campbell E, Lynch S, Suckling J, Powis S. Aberrant NF-kappaB expression in 442 autism spectrum condition: a mechanism for neuroinflammation. Mol Psychiatry 2011; 2: 27.

$44321 \quad$ Posner JB, Plum F. Spinal-Fluid pH and Neurologic Symptoms in Systemic Acidosis. $N$ 444 Engl JMed 1967; 277: 605-613.

44522 Narins RG, Emmett M. Simple and mixed acid-base disorders: a practical approach. 446 Medicine (Baltimore) 1980; 59: 161-187.

$44723 \quad$ Kraut JA, Madias NE. Lactic Acidosis. N Engl J Med 2014; 371: 2309-2319.

44824 Chan MK, Tsang TM, Harris LW, Guest PC, Holmes E, Bahn S. Evidence for disease and 449 antipsychotic medication effects in post-mortem brain from schizophrenia patients. Mol Psychiatry $450 \quad 2011 ; 16: 1189-1202$.

$45125 \quad$ Vawter M, Tomita H, Meng F, Bolstad B, Li J, Evans S et al. Mitochondrial-related gene 452 expression changes are sensitive to agonal-pH state: implications for brain disorders. $\mathrm{Mol}$ 453 Psychiatry 2006; 11: 615-679.

45426 Tomita H, Vawter MP, Walsh DM, Evans SJ, Choudary PV, Li J et al. Effect of agonal 455 and postmortem factors on gene expression profile: quality control in microarray analyses of 456 postmortem human brain. Biol Psychiatry 2004; 55: 346-352.

45727 Li JZ, Vawter MP, Walsh DM, Tomita H, Evans SJ, Choudary PV et al. Systematic 458 changes in gene expression in postmortem human brains associated with tissue $\mathrm{pH}$ and terminal 459 medical conditions. Hum Mol Genet 2004; 13: 609-616. 

correlation with white matter hyperintensity. Eur Arch Psychiatry Clin Neurosci 1998; 248: 301463306.

46429 Stork C, Renshaw PF. Mitochondrial dysfunction in bipolar disorder: evidence from magnetic resonance spectroscopy research. Mol Psychiatry 2005; 10: 900-919.

30 Hagihara H, Takao K, Walton NM, Matsumoto M, Miyakawa T, Hagihara H et al.

Immature dentate gyrus: an endophenotype of neuropsychiatric disorders. Neural Plast Neural Plast 2013; 2013, 2013: e318596.

$46931 \quad$ Hagihara H, Nakamura HK, Toyama K, Graef IA, Crabtree GR, Miyakawa T.

470 Forebrain-specific calcineurin deficiency causes immaturity of the dentate granule cells in adult 471 mice. SfN Meet 2011 Abstr.

47232 Hagihara H, Hattori S, Takamiya Y, Huang FL, Huang K-P, Miyakawa T. Neurogranin 473 deficiency causes neuronal immaturity in the dentate gyrus and frontal cortex of adult mice. SfN 474 Meet 2016 Abstr.

47533 Katayama Y, Nishiyama M, Shoji H, Ohkawa Y, Kawamura A, Sato T et al. CHD8 476 haploinsufficiency results in autistic-like phenotypes in mice. Nature 2016.

477 doi:10.1038/nature19357.

47834 Takao K, Kobayashi K, Hagihara H, Ohira K, Shoji H, Hattori S et al. Deficiency of 479 Schnurri-2, an MHC enhancer binding protein, induces mild chronic inflammation in the brain and 480 confers molecular, neuronal, and behavioral phenotypes related to schizophrenia.

481 Neuropsychopharmacology 2013; 38: 1409-1425.

48235 Zeng H, Chattarji S, Barbarosie M, Rondi-Reig L, Philpot BD, Miyakawa T et al.

483 Forebrain-specific calcineurin knockout selectively impairs bidirectional synaptic plasticity and 484 working/episodic-like memory. Cell 2001; 107: 617-629.

48536 Miyakawa T, Leiter LM, Gerber DJ, Gainetdinov RR, Sotnikova TD, Zeng H et al. 486 Conditional calcineurin knockout mice exhibit multiple abnormal behaviors related to schizophrenia. 487 Proc Natl Acad Sci U S A 2003; 100: 8987-8992.

48837 Suh J, Foster DJ, Davoudi H, Wilson MA, Tonegawa S. Impaired hippocampal 489 ripple-associated replay in a mouse model of schizophrenia. Neuron 2013; 80: 484-493.

49038 Cottrell JR, Levenson JM, Kim SH, Gibson HE, Richardson KA, Sivula M et al. Working 491 memory impairment in calcineurin knock-out mice is associated with alterations in synaptic vesicle 
cycling and disruption of high-frequency synaptic and network activity in prefrontal cortex. $J$

493 Neurosci 2013; 33: 10938-10949.

49439 Pak JH, Huang FL, Li J, Balschun D, Reymann KG, Chiang C et al. Involvement of 495 neurogranin in the modulation of calcium/calmodulin-dependent protein kinase II, synaptic 496 plasticity, and spatial learning: A study with knockout mice. Proc Natl Acad Sci 2000; 97: 1123249711237.

$49840 \quad$ Huang FL, Huang K-P. Methylphenidate improves the behavioral and cognitive deficits 499 of neurogranin knockout mice. Genes Brain Behav 2012; 11: 794-805.

$50041 \quad$ Huang FL, Huang K-P, Wu J, Boucheron C. Environmental Enrichment Enhances

501 Neurogranin Expression and Hippocampal Learning and Memory But Fails to Rescue the 502 Impairments of Neurogranin Null Mutant Mice. J Neurosci 2006; 26: 6230-6237.

$50342 \quad$ Yamasaki N, Maekawa M, Kobayashi K, Kajii Y, Maeda J, Soma M et al. Alpha-CaMKII 504 deficiency causes immature dentate gyrus, a novel candidate endophenotype of psychiatric 505 disorders. Mol Brain 2008; 1: 6.

50643 Hagihara H, Horikawa T, Nakamura HK, Umemori J, Shoji H, Kamitani Y et al. 507 Circadian gene circuitry predicts hyperactive behavior in a mood disorder mouse model. Cell Rep $508 \quad 2016 ; 14: 2784-2796$.

$50944 \quad$ Fillman SG, Cloonan N, Catts VS, Miller LC, Wong J, McCrossin T et al. Increased 510 inflammatory markers identified in the dorsolateral prefrontal cortex of individuals with 511 schizophrenia. Mol Psychiatry 2013; 18: 206-214.

51245 Catts VS, Catts SV, Fernandez HR, Taylor JM, Coulson EJ, Lutze-Mann LH. A 513 microarray study of post-mortem mRNA degradation in mouse brain tissue. Mol Brain Res 2005; 514 138: $164-177$.

$51546 \quad$ Fukuda S, Yamasaki Y, Iwaki T, Kawasaki H, Akieda S, Fukuchi N et al.

516 Characterization of the biological functions of a transcription factor, c-myc intron binding protein 1 517 (MIBP1). J Biochem (Tokyo) 2002; 131: 349-357.

$51847 \quad$ Purcell SM, Wray NR, Stone JL, Visscher PM, O’Donovan MC, Sullivan PF et al. 519 Common polygenic variation contributes to risk of schizophrenia and bipolar disorder. Nature 520 2009; 460: 748-752.

$52148 \quad$ Shi Y, Li Z, Xu Q, Wang T, Li T, Shen J et al. Common variants on 8p12 and 1q24.2 522 confer risk of schizophrenia. Nat Genet 2011; 43: 1224-1227.

52349 Stefansson H, Ophoff RA, Steinberg S, Andreassen OA, Cichon S, Rujescu D et al. 
524 Common variants conferring risk of schizophrenia. Nature 2009; 460: 744-747.

$52550 \quad$ Winder DG, Sweatt JD. Roles of serine/threonine phosphatases in hippocampel synaptic 526 plasticity. Nat Rev Neurosci 2001; 2: 461-474.

$52751 \quad$ Gerber DJ, Hall D, Miyakawa T, Demars S, Gogos JA, Karayiorgou M et al. Evidence for 528 association of schizophrenia with genetic variation in the $8 \mathrm{p} 21.3$ gene, PPP3CC, encoding the 529 calcineurin gamma subunit. Proc Natl Acad Sci U S A 2003; 100: 8993-8.

$53052 \quad$ Liu YL, Fann CSJ, Liu CM, Chang CC, Yang WC, Hung SI et al. More evidence 531 supports the association of PPP3CC with schizophrenia. Mol Psychiatry 2007; 12: 966-974.

$53253 \quad$ Sacchetti E, Scassellati C, Minelli A, Valsecchi P, Bonvicini C, Pasqualetti P et al. 533 Schizophrenia susceptibility and NMDA-receptor mediated signalling: an association study 534 involving 32 tagSNPs of DAO, DAOA, PPP3CC, and DTNBP1genes. BMC Med Genet 2013; 14: 53533.

$53654 \quad$ Eastwood SL, Burnet PWJ, Harrison PJ. Decreased hippocampal expression of the 537 susceptibility gene PPP3CC and other calcineurin subunits in schizophrenia. Biol Psychiatry 2005; 538 57: 702-710.

$53955 \quad$ Wada A, Kunii Y, Ikemoto K, Yang Q, Hino M, Matsumoto J et al. Increased ratio of 540 calcineurin immunoreactive neurons in the caudate nucleus of patients with schizophrenia. Prog 541 Neuropsychopharmacol Biol Psychiatry 2012; 37: 8-14.

$54256 \quad$ Schizophrenia Working Group of the Psychiatric Genomics Consortium. Biological 543 insights from 108 schizophrenia-associated genetic loci. Nature 2014; 511: 421-427.

54457 Ament SA, Szelinger S, Glusman G, Ashworth J, Hou L, Akula N et al. Rare variants in 545 neuronal excitability genes influence risk for bipolar disorder. Proc Natl Acad Sci 2015; 112: 35765463581.

$54758 \quad$ Xing G, Russell S, Hough C, O’Grady J, Zhang L, Yang S et al. Decreased prefrontal 548 CaMKII alpha mRNA in bipolar illness. Neuroreport 2002; 13: 501-505.

$54959 \quad$ Le-Niculescu H, Patel S d., Bhat M, Kuczenski R, Faraone S v., Tsuang M t. et al.

550 Convergent functional genomics of genome-wide association data for bipolar disorder:

551 Comprehensive identification of candidate genes, pathways and mechanisms. Am J Med Genet B 552 Neuropsychiatr Genet 2009; 150B: 155-181.

$55360 \quad$ Mertens J, Wang Q-W, Kim Y, Yu DX, Pham S, Yang B et al. Differential responses to 554 lithium in hyperexcitable neurons from patients with bipolar disorder. Nature 2015; 527: 95-99. $55561 \quad$ Neale BM, Kou Y, Liu L, Ma'ayan A, Samocha KE, Sabo A et al. Patterns and rates of 
556 exonic de novo mutations in autism spectrum disorders. Nature 2012; 485: 242-245.

55762 O’Roak BJ, Vives L, Fu W, Egertson JD, Stanaway IB, Phelps IG et al. Multiplex

558 Targeted Sequencing Identifies Recurrently Mutated Genes in Autism Spectrum Disorders. Science 559 2012; 338: 1619-1622.

$56063 \quad$ O’Roak BJ, Vives L, Girirajan S, Karakoc E, Krumm N, Coe BP et al. Sporadic autism 561 exomes reveal a highly interconnected protein network of de novo mutations. Nature 2012; 485 : 562 246-250.

56364 Talkowski ME, Rosenfeld JA, Blumenthal I, Pillalamarri V, Chiang C, Heilbut A et al. 564 Sequencing Chromosomal Abnormalities Reveals Neurodevelopmental Loci that Confer Risk 565 across Diagnostic Boundaries. Cell 2012; 149: 525-537.

$566 \quad 65 \quad$ Hagihara H, Toyama K, Yamasaki N, Miyakawa T. Dissection of hippocampal dentate 567 gyrus from adult mouse. J Vis Exp 2009. doi:10.3791/1543.

56866 Demetrius LA, Simon DK. An inverse-Warburg effect and the origin of Alzheimer's 569 disease. Biogerontology 2012; 13: 583-594.

$57067 \quad$ Wemmie JA. Neurobiology of panic and pH chemosensation in the brain. Dialogues Clin 571 Neurosci 2011; 13: 475-483.

57268 Huang L, Zhao S, Lu W, Guan S, Zhu Y, Wang J-H. Acidosis-Induced Dysfunction of 573 Cortical GABAergic Neurons through Astrocyte-Related Excitotoxicity. PLOS ONE 2015; 10: $574 \mathrm{e} 0140324$.

57569 Goldman SA, Pulsinelli WA, Clarke WY, Kraig RP, Plum F. The Effects of Extracellular 576 Acidosis on Neurons and Glia In Vitro. J Cereb Blood Flow Metab Off J Int Soc Cereb Blood Flow 577 Metab 1989; 9: 471-477.

$57870 \quad$ Nakazawa K, Zsiros V, Jiang Z, Nakao K, Kolata S, Zhang S et al. GABAergic 579 interneuron origin of schizophrenia pathophysiology. Neuropharmacology 2012; 62: 1574-1583.

$58071 \quad$ Bartzokis G. Brain myelination in prevalent neuropsychiatric developmental disorders. 581 Adolesc Psychiatry 2005; 29: 55-96.

58272 Dager SR, Friedman SD, Parow A, Demopulos C, Stoll AL, Lyoo IK et al. Brain 583 metabolic alterations in medication-free patients with bipolar disorder. Arch Gen Psychiatry 2004; 584 61: 450-458.

58573 das Neves Duarte JM, Kulak A, Gholam-Razaee MM, Cuenod M, Gruetter R, Do KQ. $586 \mathrm{~N}$-acetylcysteine normalizes neurochemical changes in the glutathione-deficient schizophrenia 587 mouse model during development. Biol Psychiatry 2012; 71: 1006-1014. 
$58874 \quad$ Nicholas MK, Lukas RV, Jafri NF, Faoro L, Salgia R. Epidermal Growth Factor

589 Receptor-Mediated Signal Transduction in the Development and Therapy of Gliomas. Am Assoc

$590 \quad$ Cancer Res 2006; 12: 7261-7270.

$59175 \quad$ Lu H, Forbes RA, Verma A. Hypoxia-inducible Factor 1 Activation by Aerobic

592 Glycolysis Implicates the Warburg Effect in Carcinogenesis. J Biol Chem 2002; 277: 23111-23115.

$59376 \quad$ Mathupala SP, Ko YH, Pedersen PL. Hexokinase-2 bound to mitochondria: Cancer's

594 stygian link to the 'Warburg effect' and a pivotal target for effective therapy. Semin Cancer Biol

595 2009; 19: 17-24.

59677 Minchenko A, Leshchinsky I, Opentanova I, Sang N, Srinivas V, Armstead V et al.

597 Hypoxia-inducible Factor-1-mediated Expression of the

598 6-Phosphofructo-2-kinase/fructose-2,6-bisphosphatase-3 (PFKFB3) Gene ITS POSSIBLE ROLE

599 IN THE WARBURG EFFECT. J Biol Chem 2002; 277: 6183-6187.

60078 Faubert B, Boily G, Izreig S, Griss T, Samborska B, Dong Z et al. AMPK Is a Negative

601 Regulator of the Warburg Effect and Suppresses Tumor Growth In Vivo. Cell Metab 2013; 17:

$602 \quad 113-124$.

60379 Pellerin L, Magistretti PJ. Glutamate uptake into astrocytes stimulates aerobic glycolysis:

604 a mechanism coupling neuronal activity to glucose utilization. Proc Natl Acad Sci 1994; 91: 1062560510629.

$60680 \quad$ Brealy JA, Shaw A, Richardson H, Singh KD, Muthukumaraswamy SD, Keedwell PA.

607 Increased visual gamma power in schizoaffective bipolar disorder. Psychol Med 2015; 45: 783-794.

$60881 \quad$ Marín O. Interneuron dysfunction in psychiatric disorders. Nat Rev Neurosci 2012; 13:

609 107-120.

61082 Sugiura Y, Honda K, Kajimura M, Suematsu M. Visualization and quantification of

611 cerebral metabolic fluxes of glucose in awake mice. Proteomics 2014; 14: 829-838.

61283 Mexal S, Berger R, Adams CE, Ross RG, Freedman R, Leonard S. Brain pH has a

613 significant impact on human postmortem hippocampal gene expression profiles. Brain Res 2006;

614 1106: $1-11$.

615

616 
Figure legends

618 Figure 1. Lower pH in the postmortem brains of patients with schizophrenia and bipolar

\section{disorder revealed by meta-analysis of publicly available data}

Box plot of $\mathrm{pH}$ in the brain of control participants (white box), patients with schizophrenia (red box)

0.027; Student's $t$-test. The boxes represent the interquartile range between first and third quartiles, the whiskers the maximum and minimum values and the circles population outliers.

Figure 2. Negative correlation between lower $\mathrm{pH}$ and increased lactate levels in the

\section{postmortem brains of mouse models of psychiatric disorders}

Bar graphs of $\mathrm{pH}(\mathbf{a})$, lactate levels (b), pyruvate levels (d), glucose levels (e), and ADT/ATP ratio 


\section{Supplementary information}

637 Supplementary Figure 1. Correlations between $\mathbf{p H}$ and lifetime antipsychotic use,

639 Scatter plots showing correlations between $\mathrm{pH}$ and lifetime antipsychotic (fluphenazine equivalents)

$0.27, P=2.1 \times 10^{-5}$; bipolar disorder: $r=0.14, P=0.085$; Total: $\left.r=0.13, P=0.0010\right)(\mathbf{c})$, and age

(controls: $r=-0.14, P=0.021$; schizophrenia: $r=-0.22, P=0.00075$; bipolar disorder: $r=0.14, P=$

0.096; Total: $r=-0.13, P=0.00092)(\mathbf{d})$. SMRI: Stanley Medical Research Institute.

645

Supplementary Figure 2. Potentially elevated glycolysis in the brains of mouse models of

648 Glycolysis-related genes whose expression was altered in the brains of mouse models of psychiatric 649 disorders were mapped in a schematic of the glycolysis pathway.

650

\section{Supplementary Table 1. Patient characteristics}

652 Antipsychotic dose (mg) is measured as fluphenazine equivalents. M, male; F, female; na, not 653 available. 
657 Genes whose expression was altered in at least four out of eight mouse datasets were processed for 658 pathway analyses.

659

660 Supplementary Table 3. Pathway analyses of the genes whose expression was altered in the 661 brains of mouse models of the psychiatric disorders using DAVID, ADGO, and GoToolBox

662 The top 20 pathways (ranked based on the $P$-value) are shown for each analysis.

663

664 Supplementary Table 4. Expression patterns of genes encoding enzymes related to glycolysis 665 pathway in the brains of mouse models of psychiatric disorders 


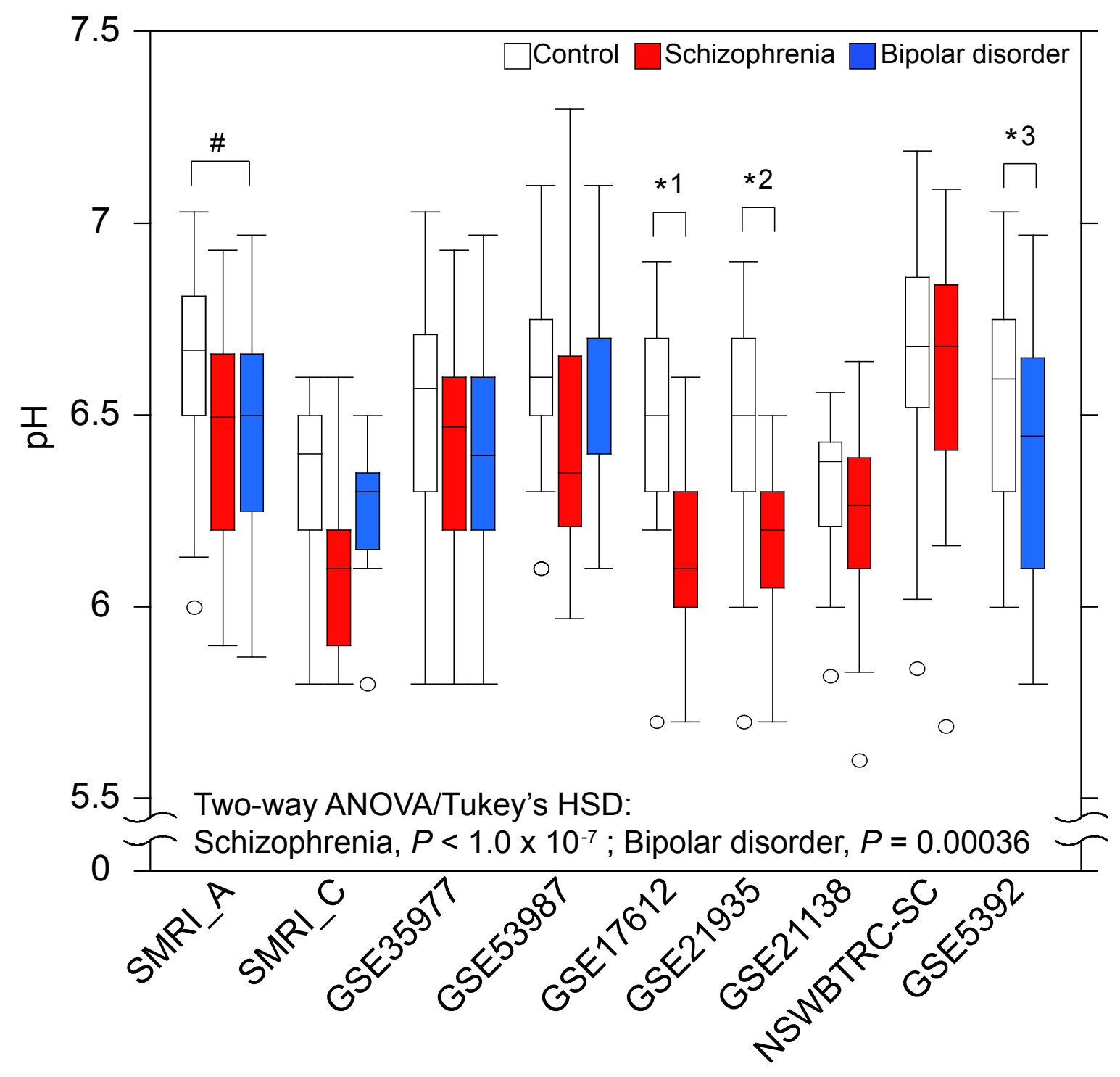

Figure 1 
bioRxiv preprint doi: https://doi.org/10.1101/083550; this version posted November 1,2016 . The copyright holder for this preprint (which was not certified by peer review) is the author/funder, who has granted bioRxiv a license to display the preprint in perpetuity. It is made available under aCC-BY-NC-ND 4.0 International license.
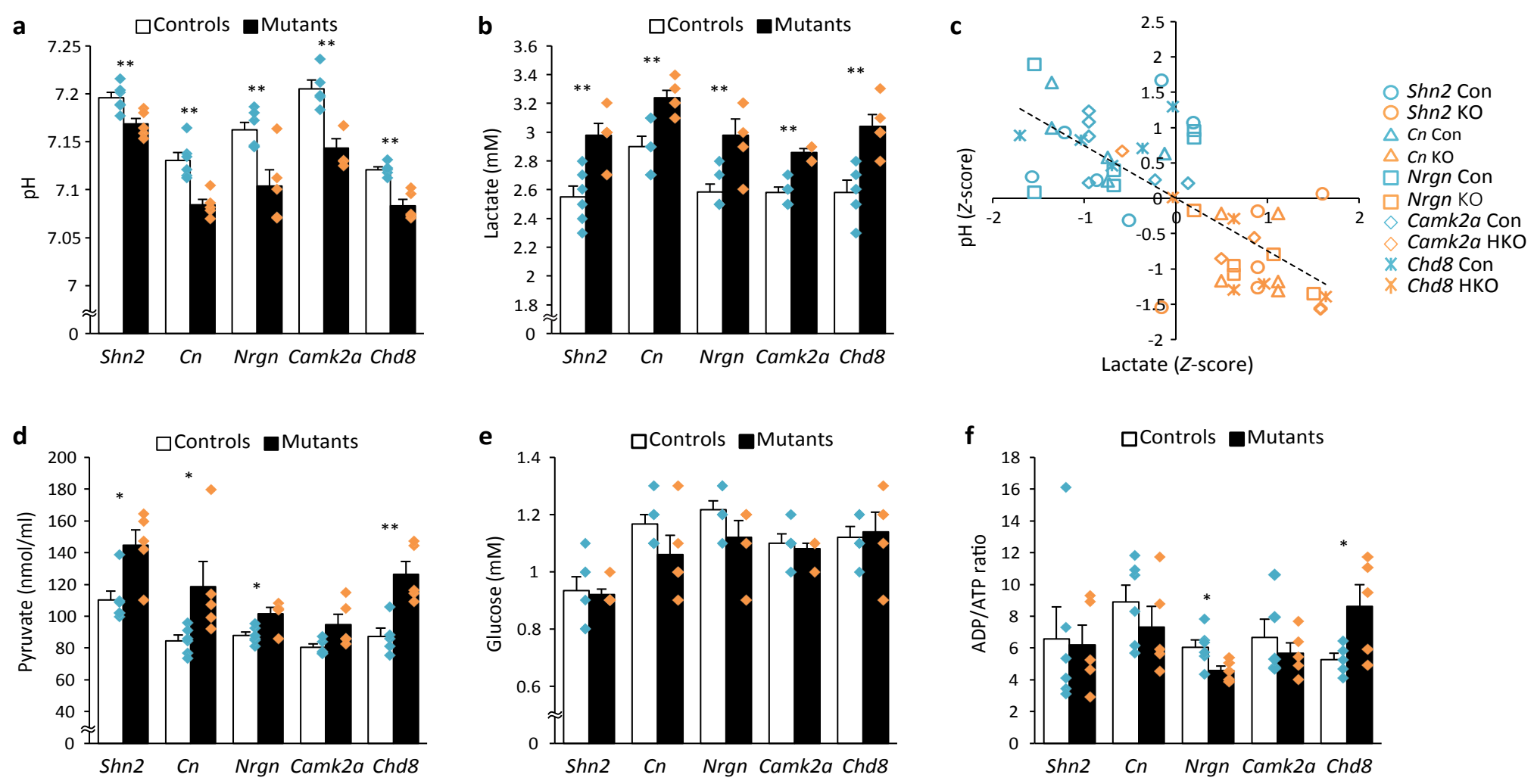

Figure 2 